\title{
A fingerprint matching hardware for smart cards
}

\author{
Sung Bum Pan ${ }^{1}$, Daesung Moon ${ }^{2}$ Kichul Kim \\ and Yongwha Chung ${ }^{4 a)}$ \\ ${ }^{1}$ Dept. of Information Control \& Instrumentation Eng., Chosun University, 375, \\ Seosuk-dong, Dong-gu, Gwangju, 501-759, Korea \\ 2 Biometrics Technology Research Team, ETRI, 161, Gajeong-dong, Yuseong-gu, \\ Daejeon, 305-350, Korea \\ 3 Dept. of Electrical \& Computer Eng., University of Seoul, 90, Jeonnong-dong, \\ Dongdaemoon-gu, Seoul, 130-743, Korea \\ ${ }^{4}$ Dept. of Computer \& Information Science, Korea University, Seochang 208, \\ Chochiwon, Chungnam, 339-700, Korea \\ a)ychungy@korea.ac.kr
}

Abstract: Using biometrics to authenticate a person's identity has several advantages over the present practices of Personal Identification Numbers or passwords. To gain maximum security in the authentication system using biometrics, the computation of the authentication as well as the store of the biometric template has to take place in a smart card. However, it is challenging to integrate biometrics into a smart card because of limited resources such as processing power and memory space. In this paper, we propose an area-time-accuracy efficient hardware design for a fingerprint matching system, which can be integrated into smart card chips. Experimental results show that the match operation can be completed in real time $(190 \mathrm{~ms})$ on the proposed hardware requiring $36 \mathrm{~K}$ gates $\left(2.4 \mathrm{~mm}^{2}\right.$ silicon area by using $0.25 \mu \mathrm{m}$ CMOS technology). Also, an Equal Error Rate (EER) of $3.8 \%$ can be obtained by using the proposed hardware solution. For the same operation, a previous software solution with a memory-efficient data structure could be executed only on a state-of-the-art, high-cost smart card and provide an EER of $6.0 \%$.

Keywords: biometrics, fingerprint verification, smart card, Matchon-Card, FPGA, hardware

Classification: Integrated circuits

\section{References}

[1] S. Prabhakar, S. Pankanti, and A. Jain, "Biometric recognition: security and privacy concerns," IEEE Security Privacy, pp. 33-42, 2003.

[2] G. Hachez, F. Koeune, and J. Quisquater, Biometrics, "Access control, smart cards: a not so simple combination," Proc. 4th Working Conf. on Smart Card Research and Advanced Applications, pp. 273-288, 2000.

[3] R. Sanchez-Reillo, J. Liu-Jimenez, and L. Entrena, "Architectures for biometric Match-on-Token solutions," LNCS, vol. 3087, pp. 195-204, 
2004.

[4] S. Yang and I. Verbauwhede, "A real time, memory efficient fingerprint verification system," Proc. ICASSP, 2004.

[5] J. Reisman, U. Uludag, and A. Ross, "Secure fingerprint matching with external registration," LNCS, vol. 3546, pp. 720-729, 2005.

[6] S. Pan, et al., "A memory-efficient fingerprint verification algorithm using a multi-resolution accumulator array for Match-on-Card," ETRI Journal, vol. 25, no. 3, pp. 179-186, 2003.

[7] T. Cucinotta, R. Brigo, and M. Di Natale, "Hybrid fingerprint matching on programmable smart cards," LNCS, vol. 3184, pp. 232-241, 2004.

[8] S. Ishida, M. Mimura, and Y. Seto, "Development of Personal Authentication Techniques Using Fingerprint Matching Embedded in Smart Cards," IEICE Trans. Inf. Syst., vol. E84-D, no. 7, pp. 812-818, 2001.

[9] A. Jain, L. Hong, and R. Bolle, "On-line fingerprint verification," IEEE Trans. Pattern Anal. Mach. Intell., vol. 19, no. 4, pp. 302-313, 1997.

[10] D. Ahn, et al., "Specification of ETRI fngerprint database," Technical Report-ETRI, 2002 (in Korean).

[11] D. Maio, et al., "FVC2004: Third Fingerprint Verification Competition," Proc. International Conference on Biometric Authentication (ICBA), pp. 1-7, 2004. [online] http://biometrics.cse.msu.edu/fvc04db/

[12] N. K. Ratha, et al., "Generating Cancelable Fingerprint Templates," IEEE Trans. Pattern Anal. Mach. Intell., vol. 29, no. 4, pp. 561-572, 2007.

\section{Introduction}

In the modern electronic world, the authentication of a person is an important task. Using biometrics to authenticate a person's identity has several advantages over the present practices of Personal Identification Numbers (PINs), passwords and smart cards that can be lost, forgotten, or stolen. In typical biometric authentication systems, the biometric templates are often stored in a central database. With the central storage of the biometric templates, there are open issues of misuse of the biometric templates such as the 'Big Brother' problem. To solve these open issues, the database can be distributed to millions of smart cards [1]. Most of the current implementations of the solution have a common characteristic that the biometric authentication process is accomplished out of the smart card introducing the risk of leaking out the biometric information. To heighten the security level, the authentication operation needs to be performed within smart cards, not in external card readers $[1,2,3,4,5,6,7,8]$. This system is called a Match-on-Card because the verification operation is executed on the smart card.

Note that standard PCs, on which typical biometric verification systems operate, have $3 \mathrm{GHz} \mathrm{CPU}$ and 512 Mbytes memory. On the contrary, a state-of-the-art smart card chip $(5 \mathrm{~mm} \times 5 \mathrm{~mm})$ can employ $50 \mathrm{MHz} \mathrm{CPU}$, 256 Kbytes of ROM program memory, 72 Kbytes of EEPROM, and 8 Kbytes of RAM at most. Since such a smart card chip has a very limited memory, typical biometric verification algorithms may not be executed even on the state-of-the-art smart card successfully. To reduce the required memory 
space significantly to be executed on a smart card, a careful analysis of the tradeoff between the memory requirement and the accuracy should be performed $[4,5]$. For example, we have developed a memory-efficient fingerprint matching algorithm by doing more computation with a special data structure [6]. However, the recognition rate of the algorithm is relatively low so that it needs to be improved further. In addition to that, another algorithm for the Match-on-Card [7] has a problem of slow execution time (i.e., $11 \sim 12$ seconds). In this paper, we present a hardware architecture for the fingerprint matching system which can be implemented on the Match-on-Card with a high recognition rate and a fast execution time.

\section{Fingerprint authentication system for the Match-on-Card}

A fingerprint authentication system has two phases: enrollment and verification. In the off-line enrollment phase, an enrolled fingerprint image is preprocessed, and the minutiae are extracted and stored. In the on-line verification phase, the "similarities" between the enrolled minutiae pattern and the input minutiae pattern are examined, and if enough similarities are found, then the two fingerprints are said to match. Note that we use a typical representation of the minutiae such as the $x$ and $y$ coordinates and the angles of the ridge endings and the bifurcations in the fingerprint image [9].

Pre-Processing refers to the refinement of the fingerprint image from a fingerprint scanner against image distortions. Extraction refers to the extraction of features in the fingerprint image. After performing these steps on the card reader, some minutiae are detected and stored into a smart card as a template file. The template file includes the position, the orientation, and the type (ridge ending or bifurcation) of the minutiae. Then, a "similarity" between the Enrolled Minutiae pattern and the Input Minutiae pattern is examined, and a decision is made by comparing that similarity and the experimentally-determined threshold in Match. Note that the Match (alignment and matching stage) is performed in the smart card, whereas the PreProcessing and the Extraction steps are executed on the card reader. Thus, it can provide greater privacy to the user because the biometric data stays in the secure environment of the smart card.

\section{Fingerprint matching hardware}

The fingerprint Match step consists of the alignment stage and the matching stage. Input to the alignment stage includes a set of enrolled minutiae $P$ and another set of minutiae $Q$ extracted from an input fingerprint image. $Q$ is transformed with a similarity transformation (rotation and translation) in the alignment stage. The result of the similarity transformation is a set of minutiae $R$. Input to the matching stage includes the enrolled minutiae $P$ and the minutiae $R$ transformed from $Q$ in the alignment stage. In the matching stage, $R$ is compared with $P$ and a matching score is computed. Eq. (1) shows notations for enrolled minutiae $P$, minutiae $Q$ extracted from the input fingerprint image and minutiae $R$ transformed from $Q$. 
In Eq. (1), $\left(p_{x}^{i}, p_{y}^{i}, p_{\theta}^{i}, p_{t}^{i}\right),\left(q_{x}^{i}, q_{y}^{i}, q_{\theta}^{i}, q_{t}^{i}\right)$ and $\left(r_{x}^{i}, r_{y}^{i}, r_{\theta}^{i}, r_{t}^{i}\right)$ represent spatial positions, orientations and types of the $i^{\text {th }}$ minutiae of each set $P, Q, R$, respectively. The number of minutiae in $P$ and $Q$ are represented by $m$ and $n$, respectively. $(\Delta x, \Delta y)$ and $\Delta \theta$ are the translation and rotation parameters, respectively. $R_{\Delta x, \Delta y, \Delta \theta}$ is decided according to the instances of $(\Delta x, \Delta y, \Delta \theta)$. $R_{\Delta x, \Delta y, \Delta \theta}$ has $n$ minutiae.

$$
\begin{aligned}
& P=\left\{\left(p_{x}^{1}, p_{y}^{1}, p_{\theta}^{1}, p_{t}^{1}\right), \cdots,\left(p_{x}^{m}, p_{y}^{m}, p_{\theta}^{m}, p_{t}^{m}\right)\right\} \\
& Q=\left\{\left(q_{x}^{1}, q_{y}^{1}, q_{\theta}^{1}, q_{t}^{1}\right), \cdots,\left(q_{x}^{n}, q_{y}^{n}, q_{\theta}^{n}, q_{t}^{n}\right)\right\} \\
& R_{\Delta x, \Delta y, \Delta \theta}=\left\{\left(r_{x}^{1}, r_{y}^{1}, r_{\theta}^{1}, r_{t}^{1}\right), \cdots,\left(r_{x}^{n}, r_{y}^{n}, r_{\theta}^{n}, r_{t}^{n}\right)\right\}
\end{aligned}
$$

In the alignment stage, the selection of reference points and the similarity transformation are performed. During the selection of reference points, two points are selected arbitrarily, one from each $P$ and $Q$. The selected points are called as reference points and represented as $\left(p_{x}^{\text {ref }}, p_{y}^{\text {ref }}, p_{\theta}^{\text {ref }}, p_{t}^{\text {ref }}\right)$ and $\left(q_{x}^{r e f}, q_{y}^{r e f}, q_{\theta}^{r e f}, q_{t}^{r e f}\right)$. During the similarity transformation, $Q$ is transformed to $R_{\Delta x, \Delta y, \Delta \theta}$ according to Eq. (3). $(\Delta x, \Delta y, \Delta \theta)$ is determined from $\left(p_{x}^{r e f}, p_{y}^{r e f}, p_{\theta}^{r e f}\right)$ and $\left(q_{x}^{r e f}, q_{y}^{r e f}, q_{\theta}^{r e f}\right)$ as shown in Eq. (2).

$$
\begin{aligned}
& \left(\begin{array}{c}
\Delta x \\
\Delta y \\
\Delta \theta
\end{array}\right)=\left(\begin{array}{c}
p_{x}^{r e f} \\
p_{y}^{r e f} \\
p_{\theta}^{r e f}
\end{array}\right)-\left(\begin{array}{c}
q_{x}^{r e f} \\
q_{y}^{r e f} \\
q_{\theta}^{r e f}
\end{array}\right) \\
& R_{\Delta x, \Delta y, \Delta \theta}\left(\begin{array}{c}
r_{x}^{i} \\
r_{y}^{i} \\
r_{\theta}^{i} \\
r_{t}^{i}
\end{array}\right)=\left(\begin{array}{cccc}
\cos \Delta \theta & -\sin \Delta \theta & 0 & 0 \\
\sin \Delta \theta & \cos \Delta \theta & 0 & 0 \\
0 & 0 & 1 & 0 \\
0 & 0 & 0 & 1
\end{array}\right)\left(\begin{array}{c}
q_{x}^{i}-q_{x}^{r e f} \\
q_{y}^{i}-q_{y}^{r e f} \\
q_{\theta}^{i} \\
q_{t}^{i}
\end{array}\right) \\
& +\left(\begin{array}{c}
q_{x}^{r e f} \\
q_{y}^{r e f} \\
0 \\
0
\end{array}\right)+\left(\begin{array}{c}
\Delta x \\
\Delta y \\
\Delta \theta \\
0
\end{array}\right) \\
& =\left(\begin{array}{cccc}
\cos \Delta \theta & -\sin \Delta \theta & 0 & 0 \\
\sin \Delta \theta & \cos \Delta \theta & 0 & 0 \\
0 & 0 & 1 & 0 \\
0 & 0 & 0 & 1
\end{array}\right)\left(\begin{array}{c}
q_{x}^{i}-q_{x}^{r e f} \\
q_{y}^{i}-q_{y}^{r e f} \\
q_{\theta}^{i} \\
q_{t}^{i}
\end{array}\right)+\left(\begin{array}{c}
p_{x}^{r e f} \\
p_{y}^{r e f} \\
\Delta \theta \\
0
\end{array}\right)
\end{aligned}
$$

The alignment stage is repeatedly performed for every possible pair of the reference points. That is, $Q$ is transformed to $R_{\Delta x, \Delta y, \Delta \theta}$ according to all possible combinations of $(\Delta x, \Delta y, \Delta \theta) . P$ is compared with every $R_{\Delta x, \Delta y, \Delta \theta}$ and, each time, the number of matching points is counted. The matching score is decided upon the maximum number of matching points.

Fig. 1 (a) shows the hardware structure of the minutiae matching system consisting of REGISTER FILE and CORE. REGISTER FILE contains registers which communicate with the microprocessor through the system bus. Because of its popularity, $A M B A A H B$ has been used as the system bus. FILE registers store the data of the Enrolled Minutiae $P$ and INPUT 
registers store the data of the Input Minutiae $Q$. PARAMETER registers provide parameter values needed for the control of the hardware. RESULT register contains the result of the match operation. CORE is composed of

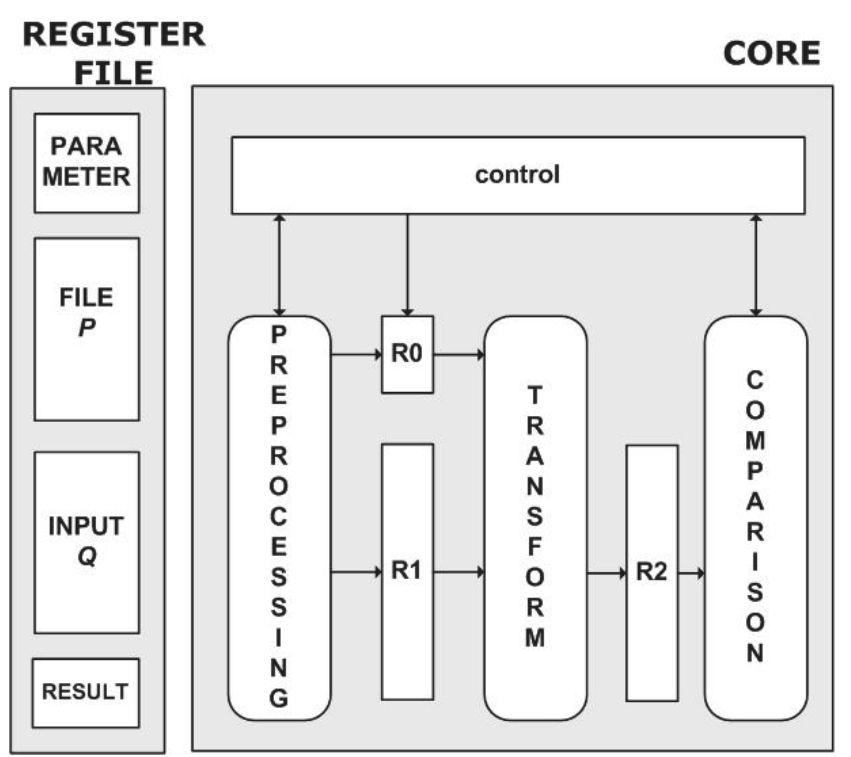

(a) Hardware architecture of matching system

OP code(select reference point or generate parameter)

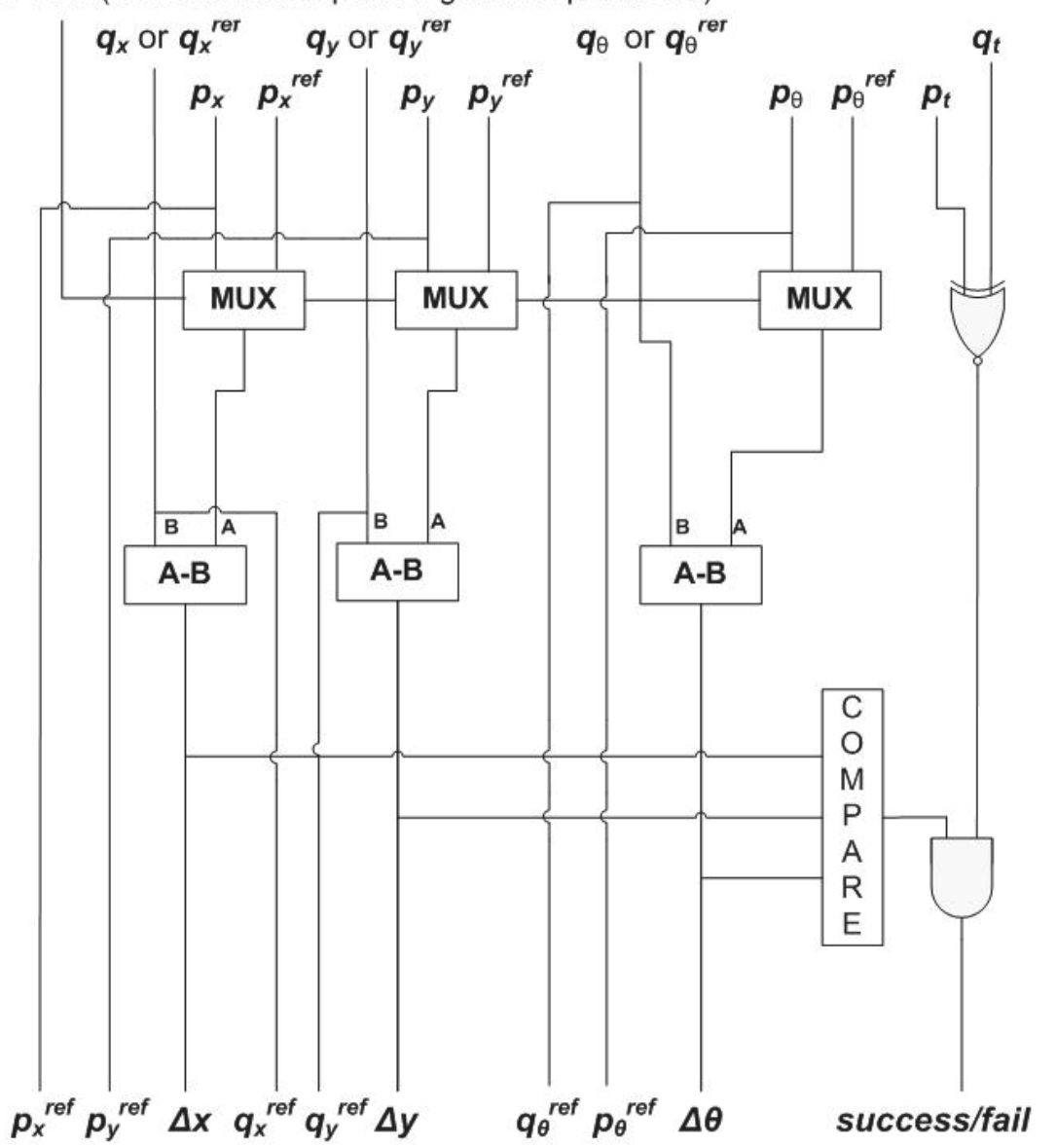




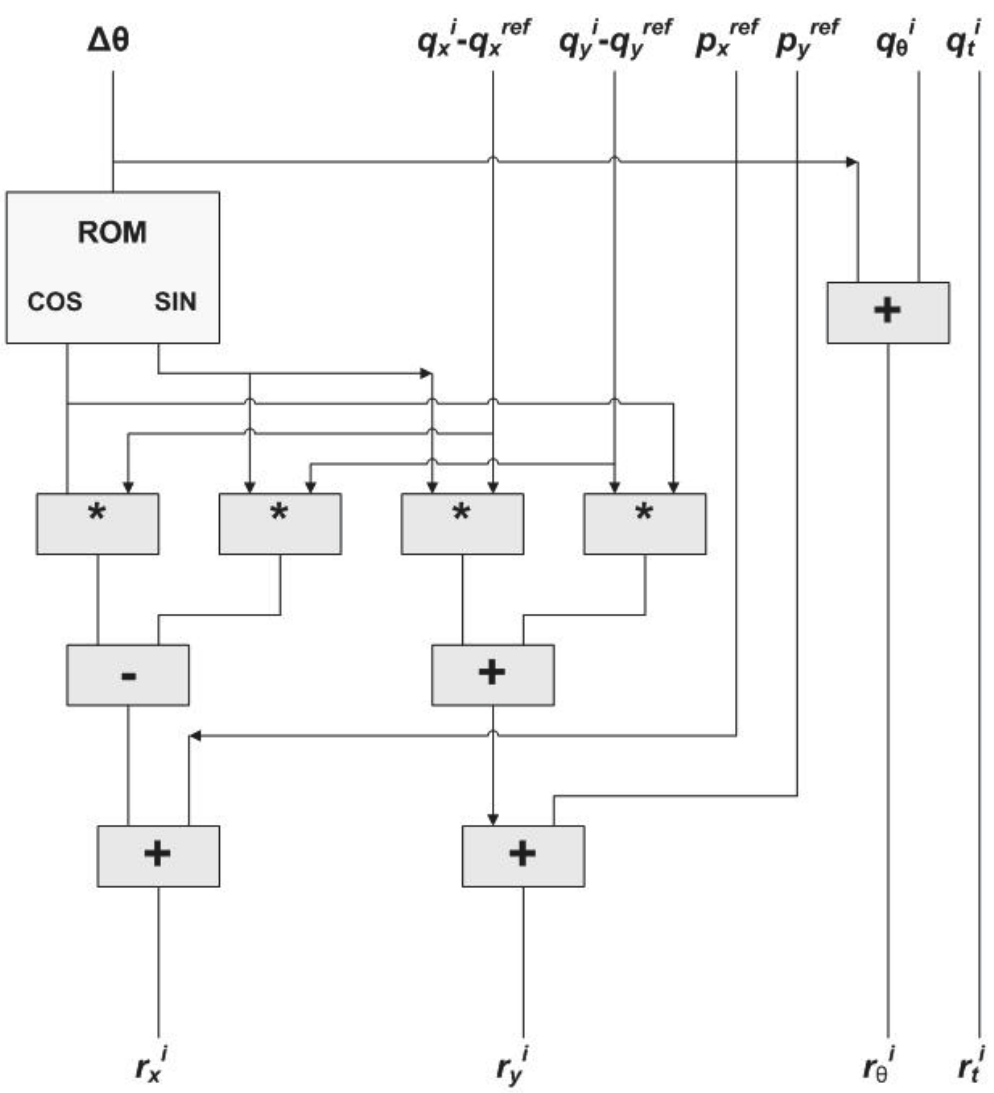

(c) TRANSFORM module

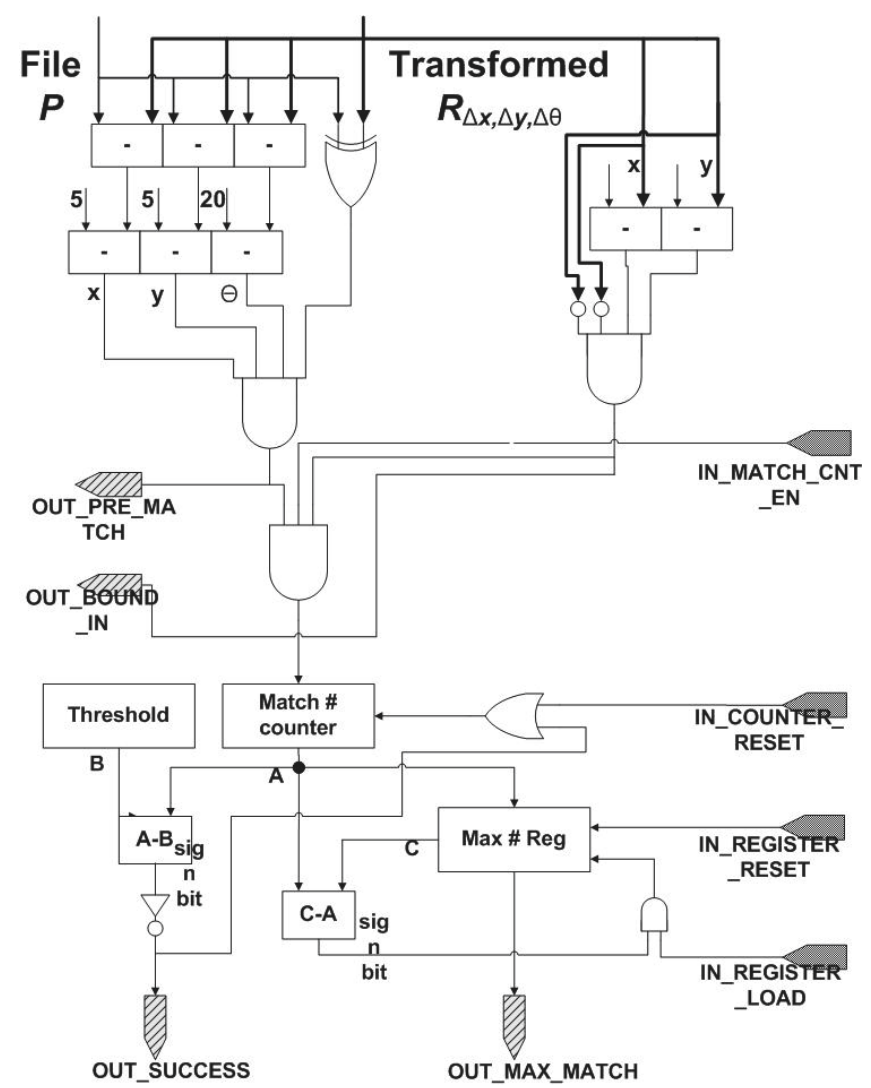

(d) COMPARISON module 
three modules - PREPROCESSING, TRANSFORM and COMPARISON. PREPROCESSING module selects reference points and generates parameters $(\Delta x, \Delta y, \Delta \theta)$ for the similarity transformation according to Eq. (2). TRANSFORM module performs the similarity transformation according to Eq. (3) with the parameters $(\Delta x, \Delta y, \Delta \theta)$. COMPARISON module computes a matching score.

The hardware structure of each module is shown in Fig. 1 (b), (c) and (d). Basic components of PREPROCESSING module are multiplexors, adders and logics for comparison. PREPROCESSING module performs two tasks. First, it selects reference points from the Enrolled Minutia $P$ and the Input Minutia $Q$, and computes the difference between the reference points to decide whether to advance for further processing with the current reference points. If the difference between the reference points exceeds a predefined threshold, further processes in the alignment stage and the matching stage are not performed. Second, it generates parameters $(\Delta x, \Delta y, \Delta \theta)$ for the similarity transformation in the alignment stage. TRANSFORM module consists of adders, multipliers and a ROM which stores trigonometric function coefficients. Because the module performs the most time-consuming computation during the minutiae matching, four multipliers are employed in parallel. TRANSFORM module transforms the Input Minutia $Q$ to the Transformed Minutia $R_{\Delta x, \Delta y, \Delta \theta}$ according to Eq. (3). COMPARISON module consists of adders, a counter, registers, and logic components for comparison. A matching score is computed by comparing positions, orientations, and types of the Enrolled Minutia $P$ and the Transformed Minutia $R_{\Delta x, \Delta y, \Delta \theta}$. The maximum value among the matching scores is stored in the RESULT register.

Note that, a pipeline methodology can be applied to improve the speed of the minutiae matching further. That is, a 2-stage pipeline can be obtained by grouping PREPROCESSING and TRANSFORM together as the first stage and making COMPARISON as the second stage. Further speedup is also possible by employing more logic components for comparison in parallel to COMPARISON module. These features are not used in the current implementation since they use more resources.

\section{Experimental results}

The minutiae matching system was implemented on Xilinx Virtex-II (XC2V2000) as shown in Fig. 2. Note that the AMBA Advanced Highperformance Bus was used to integrate it into smart card chips easily. We have evaluated our proposed hardware system using the ETRI database [10] and FVC 2004 DB set 1 [11]. The ETRI database composed of four fingerprint images per finger was collected from 1,068 individuals by using the optical fingerprint sensor [9]. The resolution of the sensor was $500 \mathrm{dpi}$, and the size of captured fingerprint images was $248 \times 292$. FVC 2004 DB set 1 consists of 8 fingerprint images per finger, after rejecting poor quality fingerprints such as [12].

Given 30 minutiae extracted from a fingerprint image using the ETRI 


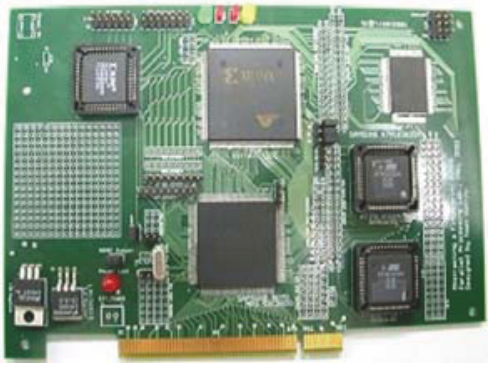

Fig. 2. The implemented minutiae matching system.

Table I. The required average area, time, and EER of the various approaches.

\begin{tabular}{|c|c|c|c|c|c|}
\hline & & $\begin{array}{c}\text { Area } \\
\left(\mathrm{mm}^{2}\right) \\
\end{array}$ & $\begin{array}{l}\text { Time } \\
(\mathrm{ms})\end{array}$ & $\begin{array}{c}\text { EER } \\
(\%) \\
\end{array}$ & $\begin{array}{c}\text { Area } \times \text { Time } \times \text { EER } \\
\left(\mathrm{mm}^{2} \times \mathrm{ms} \times \%\right)\end{array}$ \\
\hline \multirow[b]{2}{*}{$\mathrm{S} / \mathrm{W}[6]$} & $\begin{array}{c}\text { Typical } \\
\text { (ETRI DB) }\end{array}$ & 195 & 500 & 3.8 & 370,500 \\
\hline & $\begin{array}{c}\text { Memory- } \\
\text { Efficient } \\
\text { (ETRI DB) }\end{array}$ & 4.4 & 900 & 6.0 & 23,760 \\
\hline \multirow{2}{*}{$\begin{array}{c}\text { Proposed } \\
\text { H/W }\end{array}$} & (ETRI DB) & \multirow{2}{*}{2.4} & \multirow{2}{*}{190} & 3.8 & 1,733 \\
\hline & $(\mathrm{FVC} 2004 \mathrm{DB})$ & & & 6.8 & 3,100 \\
\hline
\end{tabular}

database [10], the matching with another 30 minutiae stored in a smart card can be completed in $190 \mathrm{msec}$ using a small area (36K gates). Note that, we fixed the maximum number of extracted minutiae from a fingerprint image as 30 because most of our fingerprint images have less than 30 minutiae. This fixed minutiae number can be increased with additional hardware.

Table I shows the comparison of Equal Error Rate (EER) between various approaches. Note that the area estimations were based on the $0.25 \mu \mathrm{m}$ CMOS technology used in typical smart card chips. A specially designed, software-based approach to reduce the RAM space [6] could perform the same operation in $900 \mathrm{msec}$ by using a $6.8 \mathrm{~KB}$ of RAM space $\left(4.4 \mathrm{~mm}^{2}\right)$. However, its error rate $(6.0 \%)$ is higher than that of the hardware design $(3.8 \%)$, and it could be executed only on a high-cost, 32-bit smart card. Note that, without sacrificing the error rate, the typical software-based approach cannot perform the same operation in a smart card chip. Especially, the $300 \mathrm{~KB}$ RAM space $\left(195 \mathrm{~mm}^{2}\right)$ of the typical software-based approach exceeds the allowable smart card chip area $\left(25 \mathrm{~mm}^{2}\right)$. Also, Table I shows the EER $(6.8 \%)$ of the proposed hardware system using FVC 2004 DB set 1.

\section{Conclusion}

In this paper, we have presented a hardware solution to a fingerprint minutiae pattern matching problem. The hardware can be easily integrated into an area-constrained smart card environment removing the possibility of leaking biometric information. To evaluate the effectiveness of the proposed hardware 
design, we compared it with software-based approaches in terms of area, time, and error rate. Compared to the software-based approaches, the proposed hardware design can provide superior performance by a factor of 14 or 213 in terms of the product of the area, the time, and the error rate.

\section{Acknowledgments}

This study was supported by research funds from Chosun University, 2006. 